\title{
AVALIAÇÃO DE ALTERAÇÕES REATIVAS E LESÕES CELULARES EM ESFREGAÇOS CERVICOVAGINAIS DE UMA UNIDADE DE SAÚDE BAIANA
}

\author{
EVALUATION OF REACTIVE CELLULAR CHANGES AND LESIONS IN PAP \\ SMEARS FROM A BAHIA HEALTH UNIT
}
Sintia Emmannuelle Andrade de Santana, Tássia Liz Araújo dos Santos, Carla Hass Meira, Berlley Silva Meira, Raphael Ferreira Queiroz

\begin{abstract}
Cervicovaginal exam or Pap smear is a roubust test that can detect the cervical cancer, as well as precursor lesions at early and treatable stages. This study evaluated the results of cervicovaginal cytological exams performed in 2013 at a health unit in southwestern Bahia, by documentary analysis. The sample totaled 878 exams, where 44.9\% were within normal limits; $41.6 \%$ with inflammation; $12.4 \%$ with atrophy associated with inflammation; $0.8 \%$ with atypical squamous cells of undetermined significance; $0.2 \%$ as a lowgrade squamous intraepithelial lesion and $0.1 \%$ as a high-grade squamous intraepithelial lesion. The actions developed in this institution, involving evaluation and control of the diagnosis of cervical cancer, are satisfactory, especially in the age group of forty years.
\end{abstract}

Keywords: Papanicolaou. Women's Health. Diagnosis. Prevalence.
Resumo

O exame cervicovaginal ou Papanicolaou é um teste simples que pode detectar o câncer de colo de útero, bem como lesões precursoras em um estágio precoce e curável. $O$ objetivo deste trabalho foi avaliar, por análise documental, os resultados dos exames citológicos cervicovaginais realizados em 2013 em uma unidade de saúde do sudoeste da Bahia. A amostra totalizou 878 exames, onde $44,9 \%$ estavam dentro dos limites de normalidade; $41,6 \%$ com inflamação; $12,4 \%$ com atrofia associada à inflamação; 0,8\% com células escamosas atípicas de significado indeterminado; 0,2\% como lesão intraepitelial escamosa de baixo-grau e $0,1 \%$ como lesão intraepitelial escamosa de alto-grau. As ações desenvolvidas nesta instituição, envolvendo a avaliação e controle do diagnóstico do câncer de colo uterino, são satisfatórias, especialmente na faixa etária de quarenta anos.

Palavras chave: Papanicolaou; Saúde da mulher; Diagnóstico; Prevalência. 
Introdução

O câncer de colo uterino corresponde a aproximadamente $15 \%$ dos casos de neoplasia que acomete mulheres em idade social e economicamente ativa. Essa doença representa a segunda causa de morte por câncer em mulheres no mundo, ocupando o primeiro lugar em vários países em desenvolvimento ${ }^{1}$.

O exame citológico cervicovaginal, também conhecido como exame de Papanicolaou ou preventivo, é uma das técnicas mais utilizadas em medicina e em saúde pública. Nos últimos cinquenta anos, com a ampliação de linhas de conduta por sociedades profissionais médicas e estabelecimento de programas organizados de rastreio seguindo recomendações da Organização Mundial de Saúde, esse exame passou a ser o elemento central na prevenção e no controle do câncer do colón de útero mundialmente ${ }^{1}$.

No Brasil, o Instituto Nacional de Câncer considera o exame preventivo como a principal estratégia para detectar lesões precursoras do câncer do colo de útero, uma vez que este tipo de câncer apresenta longo período de evolução, com lesões características que podem ser diagnosticadas em fase inicial, conferindo-lhe um maior potencial de prevenção e cura entre todos os tipos de câncer. Nos países desenvolvidos e nas capitais brasileiras, observou-se a redução na mortalidade pelo câncer do colo de útero, por meio da implantação de programas de rastreamento mediante a execução do exame de Papanicolaou ${ }^{2}$.

O estudo citológico do esfregaço cervical permite a identificação de um conjunto de alterações classificadas, de acordo com a presença e o grau das atipias celulares. Pelo Sistema de Bethesda, criado em 1998, essas se dividem em alterações com critérios citológicos definidos (inflamatórias, lesão intra-epitelial de baixo grau, lesão intra-epitelial de alto grau, carcinoma invasor) e alterações indeterminadas, que são aquelas que não apresentam critérios morfológicos para serem classificadas definitivamente como reativas ou como neoplásicas, denominadas mundialmente como atipias escamosas de significado indeterminado (ASCUS)3.

Todavia, as alterações anteriormente classificadas como ASCUS, numa revisão realizada no Sistema de Bethesda, em 2001, adotada pela Sociedade Brasileira de Citopatologia, a partir de 2002, as atipias de células escamosas foram subdivididas em duas categorias: células escamosas atípicas de significado indeterminado possivelmente não neoplásico (ASC-US) e células escamosas atípicas de significado indeterminado não podendo afastar lesão de alto grau (ASC-H) ${ }^{4}$.

Embora a efetividade do exame preventivo e a longa fase detectável pré-clínica do câncer de colo uterino façam com que o diagnóstico precoce seja a melhor estratégia para sua prevenção, em nossa região, os estudos epidemiológicos sobre a população de mulheres que possuem algum tipo de atipia escamosa na fase inicial são insuficientes, dificultando a definição de protocolos de acompanhamento às pacientes.

Nesse contexto, a presente pesquisa se propôs avaliar as alterações reativas e lesões celulares em esfregaços cervicovaginais dos exames de Papanicolaou realizados numa unidade de saúde responsável por parte dos exames cervicovaginais no município de Jequié, Bahia, durante o ano de 2013.

\section{Metodologia}

Neste trabalho de caráter quantitativo, descritivo, transversal, por análise documental coletou-se informações referentes aos resultados de exames citológicos cervicovaginais realizados no ano de 2013 , constantes no livro de protocolo de uma unidade de saúde responsável por parte dos exames cervicovaginais do município de Jequié, Bahia.

Foram avaliados 888 laudos de mulheres que procuraram espontaneamente a unidade de saúde no ano de 2013. Os resultados dos exames citológicos foram classificados de acordo com a última proposta de nomenclatura para laudos cervicais emitidos por laboratórios brasileiros ${ }^{4}$. Foram considerados sem alterações citológicas os resultados de exames classificados como Dentro dos Limites da Normalidade. Resultados de Inflamação e Atrofia Associada à Inflamação foram classificados como alterações celulares benignas. ASCUS (Células Escamosas Atípicas de Significado Indeterminado), LSIL (Lesão IntraEpitelial Escamosa de Baixo-Grau) e HSIL (Lesão Intraepitelial Escamosa de Alto-Grau) foram categorizados como atipias celulares. Laudos provenientes de material insatisfatório foram excluídos da análise.

Os dados foram tabulados no programa Microsoft Excel 2010 e analisados por estatística descritiva. Estes foram testados quanto à distribuição normal pelo teste de KolmogorovSmirnov no intervalo de confiança de $95 \%$. Os resultados dos exames citológicos foram 
correlacionados com os grupos etários utilizando testes de Correlações de Pearson para os dados paramétricos e Spearman para os não paramétricos, ambos de duas caudas e com nível de significância de 5\%. As análises estatísticas foram realizadas no programa GraphPad Prisma demo free (versão 6.04, GraphPad Software, Inc., CA. USA).

O estudo foi aprovado pelo Comitê de Ética em Pesquisa da Universidade Estadual do Sudoeste da Bahia (UESB), sob número de CAAE 24814114.3.0000.0055 e parecer 576.621 e respeitou as exigências formais contidas nas normas nacionais e internacionais regulamentadoras de pesquisas envolvendo seres humanos.

\section{Resultados e Discussões}

Em 2013 foram atendidas 888 pacientes na Santa Casa de Misericórdia São Judas Tadeu, em Jequié, no sudoeste da Bahia, para realização de exames citológicos cervicovaginais. A idade média das mulheres assistidas pela Instituição para realização do exame preventivo foi de $39 \pm 14$ anos, cujas moda e mediana foram de 33 e 39 anos, respectivamente. Além disso, esses resultados possuem distribuição normal de acordo com o teste de Kolmogorov-Smirnov ( $p>0,10)$.

Vale salientar que dentre os exames de Papanicolaou realizados pela Instituição, apenas $1,1 \%(n=10)$ foram considerados com material insatisfatório sendo, portanto, excluídos da análise da presente pesquisa. As pacientes com faixa etária de 15-19, 20-29, 30-39, 40-59 e $\geq 60$ anos representaram 4,6, 19,1, 27,3, 25,1, 14,9 e $9,0 \%$ das coletas satisfatórias. Notou-se que mais de $50 \%$ dos laudos pertenciam a mulheres com idade entre 30 e 49 anos.

A frequência de resultados de exames citológicos cervicovaginais sem alterações celulares totalizaram 44,9\% $(n=395) \quad($ Tabela 1). Em paralelo, a frequência de amostras na qual identificou-se inflamação e inflamação associada à atrofia correspondeu a mais da metade dos exames realizados em 2013 (Tabela 2).

Tabela 1. Distribuição etária dos exames citológicos cervicovaginais sem alterações celulares. Jequié/BA. 2014

\begin{tabular}{cc}
\hline Faixa etária (anos) & DLN \\
& $\mathbf{n}(\%)$ \\
\hline $15-19$ & $21(2,4)$ \\
$20-29$ & $74(8,4)$ \\
$30-39$ & $141(16,1)$ \\
$40-49$ & $112(12,7)$ \\
$50-59$ & $38(4,3)$ \\
$\geq 60$ & $9(1,0)$ \\
Total & $395(44,9)$ \\
\hline
\end{tabular}

$\mathrm{n}$ = número de resultados com o referido diagnóstico; DLN = Dentro dos Limites de Normalidade.

Tabela 2. Distribuição etária dos exames citológicos cervicovaginais com alterações celulares benignas. Jequié/BA. 2014

\begin{tabular}{ccc}
\hline Faixa etária (anos) & $\begin{array}{c}\text { INF } \\
\mathbf{n}(\%)\end{array}$ & $\begin{array}{c}\text { ATF + INF } \\
\mathbf{n}(\%)\end{array}$ \\
\hline $15-19$ & $19(2,2)$ & ND \\
$20-29$ & $88(10,0)$ & $4(0,4)$ \\
$30-39$ & $93(10,7)$ & $2(0,2)$ \\
$40-49$ & $102(11,6)$ & $5(0,6)$ \\
$50-59$ & $45(5,1)$ & $46(5,3)$ \\
$\geq 60$ & $18(2,0)$ & $52(5,9)$ \\
Total & $365(41,6)$ & $109(12,4)$ \\
\hline
\end{tabular}

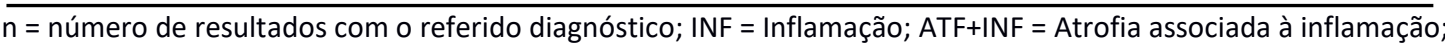
ND = Não detectado 
Os resultados também indicaram a presença de atipias celulares do tipo ASCUS, LSIL e HSIL no esfregaço citológico de $1,1 \%$ dos laudos analisados (Tabela 3). Digno de nota é que tais alterações não foram encontradas em mulheres com idade menor que 20 anos. No entanto, a identificação de ASCUS foi maior $(0,8 \%)$ que LSIL e HSIL, sendo a faixa etária entre 20 e 39 anos a mais acometida, representando $75 \%$ do total de casos para esta atipia (Tabela 3)

Tabela 3 - Distribuição etária dos exames citológicos cervicovaginais com atipias celulares. Jequié/BA. 2014

\begin{tabular}{|c|c|c|c|}
\hline Faixa etária (anos) & $\begin{array}{l}\text { ASCUS } \\
\text { n (\%) }\end{array}$ & $\begin{array}{l}\text { LSIL } \\
\text { n (\%) }\end{array}$ & $\begin{array}{l}\text { HSIL } \\
\qquad \text { n (\%) }\end{array}$ \\
\hline $15-19$ & ND & ND & ND \\
\hline $20-29$ & $2(0,3)$ & ND & ND \\
\hline $30-39$ & $2(0,3)$ & $1(0,1)$ & $1(0,1)$ \\
\hline $40-49$ & $1(0,1)$ & ND & ND \\
\hline $50-59$ & $1(0,1)$ & $1(0,1)$ & ND \\
\hline$\geq 60$ & ND & ND & ND \\
\hline Total & $6(0,8)$ & $2(0,2)$ & $1(0,1)$ \\
\hline
\end{tabular}

As lesões intraepiteliais de baixo-grau (LSIL) foram diagnosticadas em duas pacientes (Tabela 3), cuja distribuição não obedeceu à normalidade $(p=0,002)$. As duas mulheres diagnosticadas com LSIL apresentavam 30 e 50 anos de idade e, portanto, não se pode determinar correlação entre alguma faixa etária e o aparecimento de LSIL $(p>0,80)$.

HSIL caracteriza uma lesão neoplásica potencialmente maligna, sendo precursora do câncer de colo de útero. Ela foi encontrada em apenas uma paciente com 39 anos de idade, correspondendo a uma frequência de 0,1\% da amostra total (Tabela 3). Estes dados também não seguiram uma distribuição normal $(p>0,0001)$, tampouco se correlacionou com alguma faixa etária $(p=0,33)$.

O número de pacientes atendidas na unidade de saúde, no ano de 2013, representa apenas $1,6 \%$ das mulheres do município, com idade maior ou igual a 15 anos, embora refira-se a $40 \%$ das mulheres jequieenses, que realizaram exame cervicovaginal em 2013, cujos dados foram incluídos no Sistema de Informação do câncer de Colo do Útero. Esse fato demonstra o relevante papel da Instituiçãona promoção da saúde da mulher no município.

As frequências supracitadas refletem uma realidade estadual (6\%) e nacional (12\%) para o número de mulheres que realizaram o exame cervicovaginal em 2013, cujos dados também foram incluídos no Sistema de Informação do Câncer de Colo do Útero. Por outro lado, essas frequências encontram-se abaixo do esperado, pois para a Organização Mundial de Saúde (World Health Organization-WHO) é possível reduzir, de 60-90\% a incidência do câncer cervical invasivo com a cobertura de no mínimo $80 \%$ da população-alvo além da garantia de diagnóstico e tratamento adequados em casos alterados ${ }^{5}$.

No que se refere à idade média das mulheres assistidas (39 \pm 14 anos), outros estudos $^{6,7,8}$ também constataram uma idade média por volta dos trinta anos. Possivelmente, essa constatação esteja relacionada com o direcionamento dado aos programas de rastreamento, priorizando-se a faixa etária entre 35 e 59 anos, pois nessa idade encontram-se as 
mulheres mais acometidas pelo papiloma vírus humano e, consequentemente, o câncer de colo uterino ${ }^{9}$. Achados referentes ao material considerado insatisfatório, que foram excluídos da pesquisa, obedecem ao padrão mínimo de qualidade exigido pela Organização PanAmericana de Saúde, a qual preconiza que o limite máximo de esfregaços com amostra insatisfatória não deva ultrapassar $5 \%$ do total de exames $^{10}$. No entanto, o Ministério da Saúde ${ }^{11}$, descreve que mais de $40 \%$ dos municípios ainda apresentam índice de coletas insatisfatórias acima de $5 \%$, associadas principalmente a problemas nas etapas de coleta e conservação das amostras.

A avaliação da adequabilidade da amostra para exame citológico cervicovaginal baseia-se na análise microscópica do material. Amostras "satisfatórias" apresentam quantidade representativa de células bem distribuídas, fixadas e coradas, de modo a facilitar o diagnóstico. Já a coleta "insatisfatória" compreende a amostra cuja leitura é prejudicada pela escassez celular, presença de hemácias, piócitos, artefatos de dessecamento, superposição celular e/ou presença de contaminantes externos ${ }^{6}$.

Os dados relativos à idade média das mulheres (entre 30 e 49 anos) que representaram mais de $50 \%$ da amostra condizem com o perfil de pacientes que realizam periodicamente o exame preventivo ${ }^{12}$. Achados epidemiológicos de outros estados brasileiros ${ }^{13,14}$, também demonstraram resultados semelhantes. De fato, o INCA demonstra que a faixa etária de 35 a 49 anos corresponde ao pico de incidência de lesões cervicovaginais pré-malignas, sendo prioridade no rastreamento do câncer do colo uterino $^{15}$.

A porcentagem dos exames categorizados como dentro do limite de normalidade (44,9\%) ainda que não seja a ideal, foi compatível com outros estudos epidemiológicos. O Instituto Nacional de Câncer caracteriza a expressão "Dentro dos Limites de Normalidade no Material Examinado" como um diagnóstico completamente normal e cuja conduta clínica é prosseguir com a rotina de rastreamento citológico. É importante esclarecer que de acordo com o Instituto Nacional de Câncer, a inclusão da expressão "no material examinado" visa a estabelecer, de forma clara e inequívoca, aspectos do material submetido ao exame ${ }^{4}$.

Dados analisados no Laboratório Central do Estado do Pará em 2008, constataram que aproximadamente $96 \%$ dos esfregaços citológicos foram considerados como dentro dos limites de normalidade ${ }^{6}$. Por outro lado, num estudo progressivo de todos os exames cervicovaginais cadastrados no Sistema de Informação do Câncer do Colo do Útero entre os anos de 2006 e 2013, cerca de 59\% dos exames realizados em todo Brasil foram normais ${ }^{15}$.

O diagnóstico de inflamação representou 62,5 a $84 \%$ dos achados em exames de Papanicalaou realizados em outras regiões do Brasil ${ }^{8,14}$, sendo que a presença de sinais e sintomas ginecológicos associados ao processo inflamatório foram os principais motivadores a levar as mulheres ao atendimento médico especializado ${ }^{16}$.

A frequência de $1,1 \%$, constatada para as atipias celulares do tipo ASCUS, LSIL e HSIL, não destoa das investigações realizadas nos estados do Pará6, Maranhão7, em Sergipe ${ }^{8}$, cujas frequências variaram de 1 a 5\%. Embora existam diferenças socioeconômicas importantes entre os grupos estudados, a variação nos resultados também pode ser reflexo de uma maior procura por atendimento médico pelas mulheres pertencentes aos últimos estudos supracitados que, por conseguinte, reflete no diagnóstico precoce de alterações celulares no esfregaço cervicovaginal.

Como constatado no presente estudo, ASCUS é a mais comum anormalidade epitelial diagnosticada nos esfregaços corados pelo método de Papanicolaou. No entanto, de acordo com são considerados aceitáveis diagnósticos de ASCUS e atipias glandulares de significado indeterminado com taxas inferiores a $5 \%$ do total de exames realizados em um laboratório ${ }^{17}$.

A frequência de ASCUS descrita nos laudos de exames das pacientes assistidas pela unidade de saúde de Jequié foi menor que o limite recomendado pelo Instituto Nacional de Câncer, bem como das frequências descritas na literatura, nas quais os valores corresponderam a 1,25$4,98 \%$ dos achados citológicos nos esfregaços cervicovaginais analisados em outros laboratórios $7,13,15$.

Os diagnósticos de LSIL, aqui verificados se encontram bem abaixo dos observados na literatura $(0,34 \text { a } 12,5 \%)^{7,18}$. As idades das mulheres com LSIL variaram de 19 a 30 anos, já aquelas com laudos de HSIL a faixa etária foi de 35 e 50 anos $^{14}$.

De maneira geral, a frequência observada de HSIL neste estudo correlacionou-se com outra investigação realizada no Maranhão7. A frequência relativamente reduzida de HSIL nestas pacientes $(0,1 \%)$, provavelmente esteja associada com o cuidado contínuo das mulheres frente aos fatores de riscos que levam o desenvolvimento 
do câncer de colo uterino a procura por atendimento precoce nas unidades de saúde, uma vez que os programas de prevenção têm como público alvo as mulheres jovens, em idade fértil.

\section{Conclusão}

Os resultados dos exames cervicovaginais realizados durante todo o ano de 2013, pela unidade saúde no município de Jequié, mantiveram-se de acordo com as recomendações e exigências do Instituto Nacional de Câncer, bem como apresentaram frequências relacionadas àquelas descritas na literatura. Um fato importante verificado neste

\section{Referências}

1. Consolaro M, Siliber S. Citologia CérvicoVaginal - Texto e Atlas. São Paulo: Roca, 2012.

2. Gonzaga CMR, Freitas-Junior $\mathrm{R}$, Barbaresco AA, Martins E, Bernardes BT, Resende AP M. Cervical cancer mortality trends in Brazil: 1980-2009. Cad Saúde Pública. 2013 mar; 29(3): 599-608.

3. Aguiar LS, Moura TPS, Etlinger D, Yamamoto LSU, Loreto C, Cury LCB, et al. Avaliação crítica das nomenclaturas diagnósticas dos exames citopatológicos cervicais utilizadas no Sistema Único de Saúde (SUS). Rev Bras Ginecol Obstet . 2011; 33(3): 144-149.

4. Ministério da Saúde (BR). Secretaria de Atenção à Saúde, Instituto Nacional de Câncer, Coordenação de Prevenção e Vigilância. Nomenclatura brasileira para laudos citopatológicos cervicais e condutas clínicas preconizadas: recomendações para profissionais de saúde. Brasília: Ministério da Saúde; 2006.

5. Organization. International Agency for Research on Cancer [Internet]. Lyon: Globocan. 2008 [acesso em 2016 mai 14]. Disponível em: http://globocan.iarc.fr/

6. Sousa MS, Canto ASS, Tsutsumi MY, Maciel MC, Zeferino LC. Perfil dos exames citológicos do colo do útero realizados no Laboratório Central do Estado do Pará, Brasil. Rev Pan-Amaz Saúde. 2011 jun; 2(2): 27-32.

7. Silva DSM, Silva AMN, Brito LMO, Gomes SRL, Nascimento MDSB, Chein MBC. Rastreamento do câncer do colo do útero no Estado do Maranhão, Brasil. Ciênc. saúde coletiva [Internet]. 2014. abr [acesso em 2016 mai 10]; 19 (4): 1163-1170. Disponível em: http://www.scielo.br/scielo.php?script=sci artte xt\&pid=S1413-81232014000401163\&Ing=en. estudo foi que as pacientes, com idade entre 35 a 49 anos, procuraram pelo atendimento médico especializado em maior proporção, sendo a faixa etária de maior risco para o desenvolvimento do câncer de colo uterino. Sendo assim, o perfil epidemiológico constatado indica que o rastreamento e controle do diagnóstico do câncer de colo do útero na referida instituição podem ser considerados satisfatórios e de grande relevância para manutenção da saúde da mulher, na comunidade local.

8. Reis NROG, Costa AMC, Madi RR, Melo CM. Perfil microbiológico e alterações citológicas associadas em material cérvico-vaginal coletado em consultório de enfermagem, de 2009 a 2011 em Aracaju/SE. Scientia Plena. 2013 mai; 9(5):1-8. 9. Oliveira-Silva M, Lordello CX, Zardo LM, Bonvicino CR, Moreira MA. Human Papillomavirus in Brazilian women with and without cervical lesions. Virology Journal [Internet]. 2011 Jan 5 [acesso em 2016 jun 02]; 8 (4). Disponível em: https://www.ncbi.nlm.nih.gov/pmc/articles/PMC 3024957/.

10. Girianelli VR, Gamarra CJ, Silva, GA. Os grandes contrastes na mortalidade por câncer do colo uterino e de mama no Brasil. Rev Saúde Pública. 2014 jun; 48(3): 459-467.

11. Ministério da Saúde (BR). Secretaria de Atenção à Saúde, Instituto Nacional de Câncer. Informativo trimestral do Instituto Nacional de Câncer. Monitoramento das ações de controle dos cânceres do colo do útero e de mama. Brasília: Ministério da Saúde; 2010.

12. Ministério da Saúde (BR), Secretaria de Atenção à Saúde, Departamento de Atenção Básica. Controle dos cânceres do colo do útero e da mama. Brasília: Ministério da Saúde; 2013.

13. Barcelos AC, Michelin MA, Adad SJ, Murta EFC. Atypical squamous cells of undetermined significance: Bethesda classification and association with Human Papillomavirus. Infect Dis Obstet Gynecol [Internet]. 2011. [acesso em 2016 jun 20]; 2011(904674). Disponível em: https://www.hindawi.com/journals/idog/2011/9 04674/

14. Costa JHG, Souza IRA, Santos EJA, Prazeres BAP, Andrade ML, Melo MFC et al. Prevenção do câncer de colo do útero em comunidades ribeirinhas atendidas pelo 
Programa Luz na Amazônia, Estado do Pará, Brasil. Rev Pan-Amaz Saude [Internet]. $2011 \mathrm{dez}$ [acesso em 2016 mai 08]; 2(4): 17-22. Disponível em:

http://scielo.iec.pa.gov.br/scielo.php?script=sci arttext\&pid=S217662232011000400003\&lng=pt. 15. Costa FAC, Longatto-Filho A, Pinheiro C, Zeferino LC, Fregnani j $\mathrm{H}$. Historical Analysis of the Brazilian Cervical Cancer Screening Program from 2006 to 2013: A Time for Reflection. Plos one [Internet]. 2015 set 24 [acesso em 2016 mar 19]; 10 (9). Disponível em: https://www.ncbi.nlm.nih.gov/pubmed/2640273 $\underline{7}$ 16. Urrutia MT, Poupin L. Women with Cervical Cancer: Perceptions about the Papanicolaou Test. Aquichán. 2015 out; 15 (4): 499-507.

17. Carvalho VF, Kerber NPC, Wachholz VA, Pohlmann FC, Marques LA, Francioni FF. Access to Papanicolaou Test by the Unified Health System users. Rev Rene. 2016 mar-abr; 17(2): 198-207.

18. Guimarães JV, Salge AKM, Oliveira FA, Lino Júnior RS, Castro ECC, Reis MA, Teixeira VPA. Freqüência de alterações cérvico-vaginais em mulheres submetidas ao exame citopatológico. Rev. Eletr. Enf. [Internet]. 2007 [acesso em 2016 jun 02]; 9 (3): 815-20. Disponível em: http://www.fen.ufg.br/revista/v9/n3/v9n3a20.ht $\underline{\mathrm{m}}$

\section{Endereço para Correspondência}

Universidade Estadual do Sudoeste da Bahia UESB

Rua, Av. José Moreira Sobrinho, s/n - Jequiezinho, Jequié - BA

CEP.: 45206-190

e-mail: rfqueiroz@uesb.edu.br

Recebido em 24/05/2018

Aprovado em 07/11/2018

Publicado em 20/12/2018 